\title{
An Exploration into Supporting Artwork Orientation in the User Interface
}

\author{
George W. Fitzmaurice, Ravin Balakrishnan, Gordon Kurtenbach, Bill Buxton \\ Alias|wavefront \\ 210 King Street East \\ Toronto, Canada, M5A $1 \mathrm{~J} 7$ \\ $<$ gf, ravin, gordo, buxton>@aw.sgi.com \\ $+1416362-9181$
}

\begin{abstract}
Rotating a piece of paper while drawing is an integral and almost subconscious part of drawing with pencil and paper. In a similar manner, the advent of lightweight pen-based computers allow digital artwork to be rotated while drawing by rotating the entire computer. Given this type of manipulation we explore the implications for the user interface to support artwork orientation. First we describe an exploratory study to further motivate our work and characterize how artwork is manipulated while drawing. After presenting some possible UI approaches to support artwork orientation, we define a new solution called a rotating user interface (RUIs). We then discuss design issues and requirements for RUIs based on our exploratory study.
\end{abstract}

KEYWORDS: rotating user interfaces, RUI, pen-based computers, GUI toolkits, tablets, LCDs, two-handed input.

\section{INTRODUCTION}

The human act of drawing or sketching is a rich technique for entering data into a computer. The notion of sketching as a means of computer input has been around as early as 1945 [4] and 1960 [14]. As display and input technologies evolve systems can be created which approach the quality and style of sketching on paper. Computers have become very adept at emulating the myriad of non-computer drawing effects and properties. For example, displays can show millions of colors and draw at high resolutions, computer "paint brushes" can simulate lead pencils, ink pens, oil brushes, air brushes. Flat panel display technologies are allowing displays to be placed flat on the desk or held in the hand. Input technologies also emulate traditional art tools. For example, computer pens can be made in the same shape as pens, pencils, or brushes and operated without batteries or a tether cord. Finally, the combination of these technologies makes it possible to produce a computer display input system which functions like a drawing tablet where one can draw directly on the display [16].

This combination of display and input surface is extremely appealing to traditional artists. First, up until recently, computer drawing was generally performed by having an input tablet which was separate from the display surface. The displacement of the display and drawing surface produces an eye-to-hand coordination problem (although highly experi-

\section{Published in Proceedings of 1999 ACM Conference on Human Factors in Com- puting Systems (CHI'99), pp 167-174.}

enced tablet and display users report that this problem can be overcome with experience). However, the problem nonetheless causes many traditional artists to avoid computer drawing. Also, for an artist switching between paper drawing and computer drawing the displacement may be irritating.

Another appealing aspect of combined display and input surfaces is that they approach the form factor of paper and are graspable user interfaces [7]. Because of this, these systems can be manipulated very much like a piece of paper: the drawer can rotate and move the system to either make themselves more comfortable or to facilitate certain kinds of drawing strokes. One need only attempt to draw a crosshatch pattern on a small piece of paper to realize that moving and rotating the piece of paper can be an integral part and almost subconscious act of drawing.

When artists work at a desk, they rotate their drawing paper while the desk top remains fixed. This arrangement has been embodied in a specialized desk called an "animator's turntable" which is the inspiration for our work. Figure 1 shows such a turntable used by an animator for creating drawings for cel based animation. The artwork which is positioned on the turntable in the center of the desk can be rotated while the "UI" (the pencil tray, desktop, etc.) remains fixed.

This type of manipulation gives rise to the following question: if the display and input surface can be rotated, does the user interface need to adapt to the rotation?

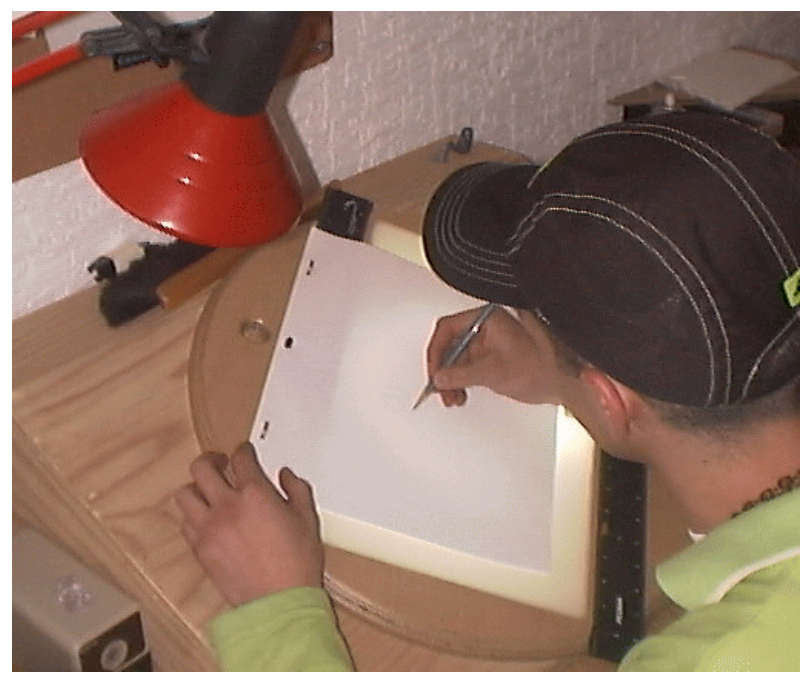

Figure 1: Animator's turntable. 
In this paper we present a study of the characteristics of how artwork is manipulated while drawing and discuss how these characteristics place new requirements on the user interface. While we propose a variety of solutions to facilitate artwork orientation, we mainly focus on introducing the concept of a rotating user interface and associated design issues.

\section{EXPLORATORY STUDY}

In order to gain insight into how and why artists rotate and manipulate their drawing surface while they work, we conducted an exploratory study. The inspiration for this study is based partially on Guiard's [8] study on paper orientation and translation for handwriting tasks and builds upon Hinckley's circle sketching study [9].

\section{Why Rotate?}

Based on traditional practices of artists and the literature, we expected to observe some amount of rotation. First, studies have been conducted on characterizing stroke orientation during simple drawing tasks and found that subjects operate within an articulation comfort range [13, 15]. These ranges are consistent when we examine the bio-mechanics of the fingers, wrist, and arm. Secondly, there are perceptual issues to be factored into the drawing process. Rotating the artwork may facilitate a drawing task simply by preventing the hand from obscuring a key area of the drawing. Finally, traditional artists working outside the computer medium are trained to factor into the drawing process the characteristics of the raw materials being used. For example, oil paint or ink will smear if an artist is not careful. Thus, the motor skills, perceptual issues, and artist's traditional practices will likely influence computer mediated drawing processes.

\section{Description of Study}

The exploratory study consisted of 3 main drawing configurations with 5 representative tasks.

\section{Conditions: Paper, Tablet and 6D}

The first condition, Paper, had artists draw on a piece of paper with a mechanical pencil on a tabletop (Figure $2 \mathrm{a}$ ). Fixed on the tabletop was a large piece of white paper. Carbon paper was laid on top of this white paper, and tissue paper laid on the carbon paper. Artists drew on a smaller piece of paper which they could freely move on top of this three layered work surface. This setup allowed us to capture the stroke patterns based on a fixed "user" orientation relative to the table (from the marks on the large white paper under the carbon paper). The paper condition served as a reference condition involving no computer technology to capture traditional drawing patterns.

The second condition, Tablet, required artists to draw on a tethered Wacom digitizing tablet [16] measuring 9.5 by 13 inches. This condition roughly reflects the computer configuration of a portable, combined display and digitizer surface (Figure 2b). The stylus was a mechanical pencil with an embedded Wacom stylus sensor. A 6 degree of freedom sensor (the Bird [1]) was attached to the top left corner of the tablet. Paper was affixed to the active digitizing surface ( 6.5 by 8.5 inches). Artists were instructed to use the tablet on the tabletop. This configuration allowed us to track both the orientation and translational manipulations of the tablet as well as track all pencil strokes.
The third condition, $6 D$, was the same as the Tablet condition except that artists were not allowed to use the tabletop. Instead they started the task with the tablet in their lap and could move the tablet in 3D space (Figure 2c).

The rationale for these three conditions was to see if the cord on the tablet and the weight/form-factor of the tablet had any impact on subjects drawing styles and manipulation of the drawing surface.
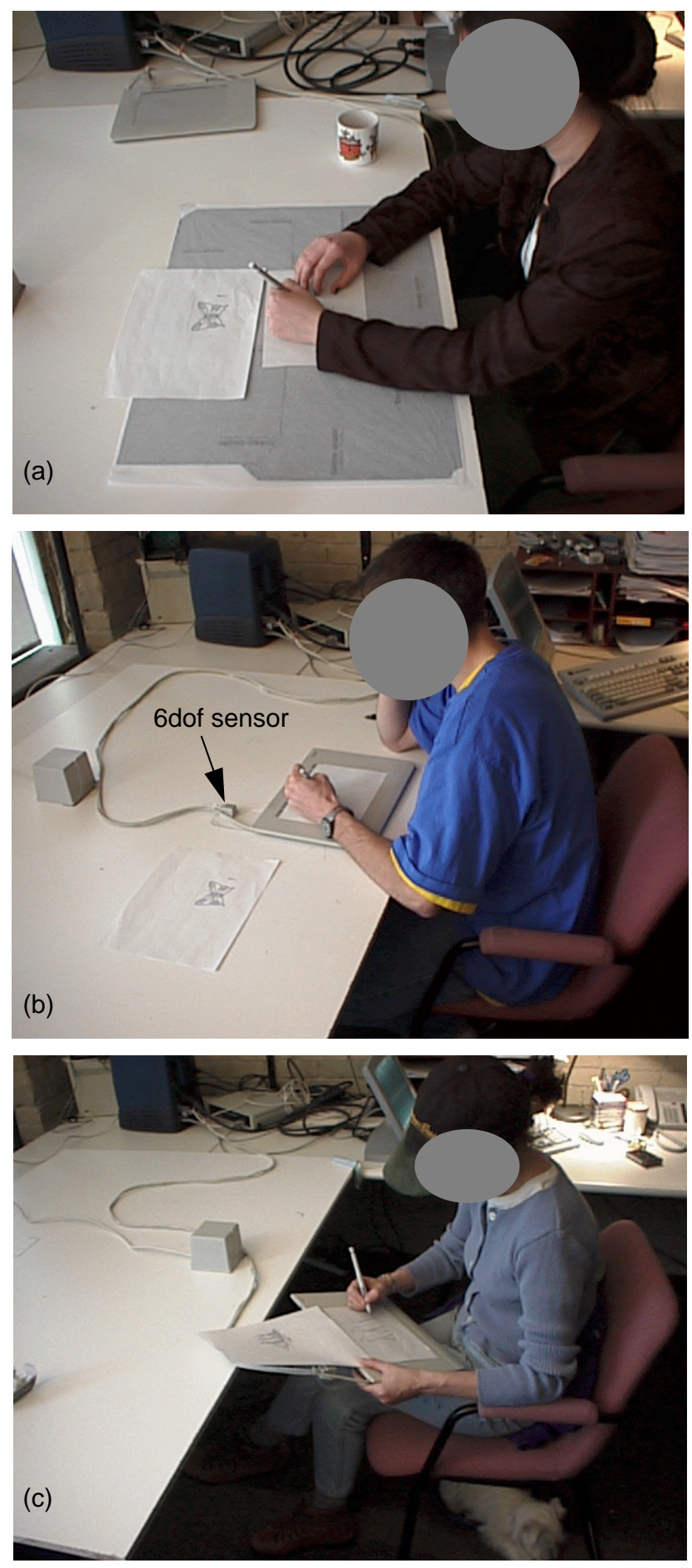

Figure 2: Three drawing conditions: (a) Paper, (b) Tablet and (c) $6 D$ conditions. 


\section{Tasks}

A total of five representative pencil tasks were defined (Figure 3). Two were handwriting tasks, three were drawing tasks. The first task, Text, asked subject to transcribe a text passage onto a sheet of $8 \times 11$ inch college ruled paper. Subjects were asked to "double-space" their handwriting to fill their page quickly. The second task, Label, required subjects to transcribe labels from a master graphical template onto an empty graphical template in a variety of orientations. The task was designed to encourage a great deal of rotation. The third and fourth task, Ship and Butterfly, asked subjects to reproduce the provided line drawings of a ship and butterfly respectively. The fifth task, Freeform, asked subjects to sketch their favorite drawing in under 5 minutes. (a)

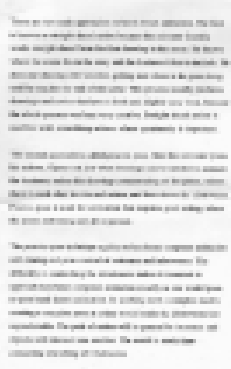

(c)

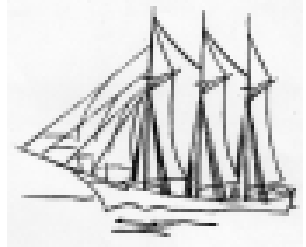

(b)

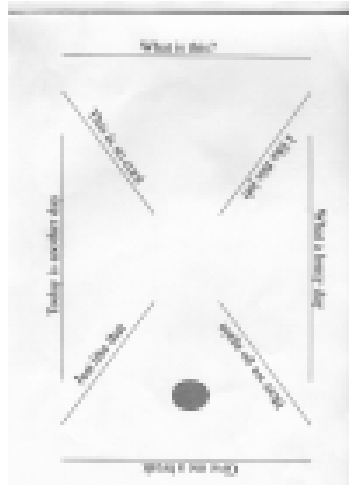

(d)

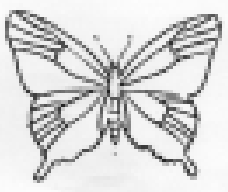

Figure 3: Pencil tasks consisting of (a) Text, (b) Label, (c) Ship, and (d) Butterfly.

All tasks were designed to take no more than five minutes to complete. Artists were told they could move and rotate the paper/tablet throughout the task.

Note that for all conditions and tasks, artists used the same mechanical pencil with integrated Wacom stylus sensor. Artists sat in a non-rotating seat to preserve their orientation relative to the drawing table. Subjects were video recorded throughout the exploratory studies.

A total of six artists participated in our exploratory study. Four were right handed, and two were left handed. They performed the five pencil tasks for all three equipment conditions. To reduce transfer effects, the conditions were presented to subjects in a completely counterbalanced order. Within each condition, the order of presentation of each task was randomized. In summary, each subject performed a total of fifteen pencil tasks taking a total of approximately ninety minutes. To minimize fatigue, subjects took short breaks between each task.

\section{Results}

As this was a holistic exploratory study, we were mainly interested in observing general patterns of behavior instead of measuring performance with rigorous metrics (e.g., speed and accuracy). Although we did record task completion times, the large individual differences in drawing style and ability we observed amongst the subjects precludes any meaningful conclusions being drawn from this timing data.

We now present separate analyses for the Text, Label, and three drawing tasks. Due to apparent large individual differences, we present views of the data for individual subjects rather than summarizing across all subjects. We then discuss the issues pertaining to the effects of the three conditions (Paper, Tablet, and 6D). Due to the difficulty in extracting data via videotape analysis, we only present detailed data for the Paper condition for the Label task.

\section{Text Task}

As shown in Figure 4, subjects demonstrated two main styles of articulation in the Text transcription task. In the first style (Figure 4a,b), subjects first oriented the paper to their preferred orientation and subsequently kept adjusting the paper with their non-dominant hand. As such, the effective working area was limited to a small band of writing (Figure 4b). This result replicates Guiard's [2, 8] observations of people's writing behavior.
Relative to Paper

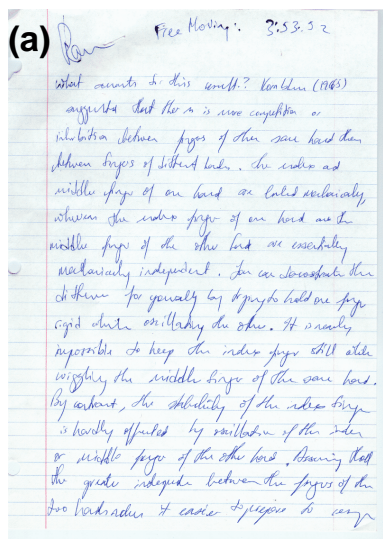

(c)

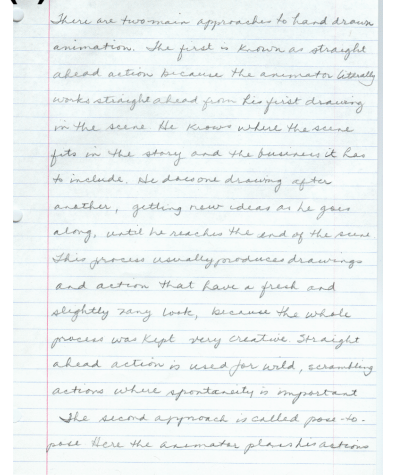

Relative to Table

(b)

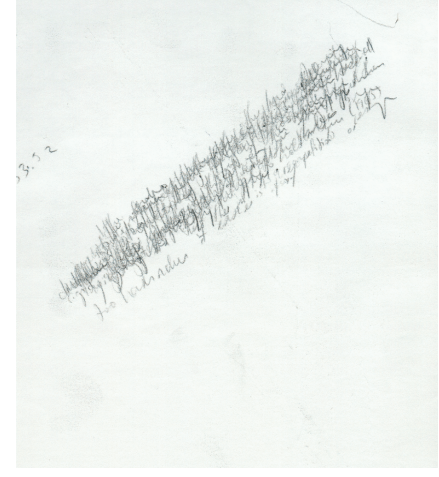

(d)

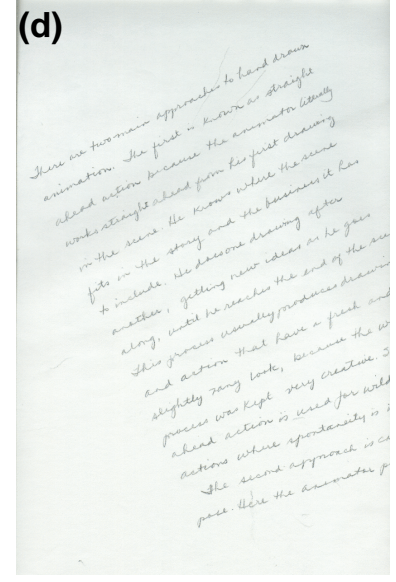

Figure 4: Sample results of exploratory study in the Paper condition with the Text task. The left column, figures (a) and (c), show the results of the text transcription output of 2 subjects. The right column, figures (b) and (d), shows the results relative to the physical table. This was captured using carbon paper as described in the "Description of Study" section of this paper. 
In the second style (Figure 4c,d), subjects only oriented the paper to their preferred orientation. Unlike the first style, they did not make substantial adjustments. As a result, they used a much larger work space. We hypothesize that this behavior is due to the nature of the transcription task which sometimes caused subjects to use the index finger of their non-dominant hand to keep their place in the source text. Their non-dominant hand is thus not available to easily reposition the writing paper.

\section{Label Task}

This task was included in our study as a "control" condition. Essentially, we wanted to observe subjects behavior when presented with a task that would be relatively hard to perform without rotating the paper (e.g., it is practically impossible to write text upside down). However, this is not a completely contrived task: architectural and engineering drawings often require that text be written at varying orientations on the page.

This is the only task where we present detailed analysis for the Paper condition. This data was derived from manual analysis of videotapes of the study.

As expected, we found that most subjects rotated the drawing surface to at least eight orientations (Figure 5). This corresponded to the need to write text in the required eight orientations (Figure 3b). The only anomaly was subject S5 who only made four major rotations in the Paper condition, but, like the other subjects, made multiple rotations in the Tablet and $6 D$ conditions. We also note that the amount of time spent at each orientation is about equal and that the rotations are well distributed across the full 360 degree range of possible rotations.

\section{The Three Drawing Tasks}

As with the Text task, subjects exhibited a range of drawing behaviors in the three drawing tasks. We showcase three common styles in Figure 6 within the Ship drawing task in the Paper condition. Similar behavior was observed in the Butterfly and Freeform tasks. In the first style (Figure 6a,b), subjects oriented the drawing surface to their preferred orientation and then kept it fixed for the duration of the task.

In the second style (Figure 6c,d), subjects oriented the drawing surface to their preferred orientation, and subsequently made a small number of adjustments to the orientation and position of the drawing surface.

In the third style (Figure 6e,f), subjects made numerous adjustments to the orientation and position of the drawing surface throughout the duration of the task.

We note that only one subject ( $\mathrm{S} 1$, and also a subject in an earlier pilot study) exhibited the third style (Figure 8). Given that this subject and the pilot subject are professional artists who work under serious time constraints on a daily basis and are perhaps trying to optimize for speed, whereas the other subjects were art school students who may have deemphasized speed, we hypothesize that the amount of rotation may be coupled to the speed of drawing. However, we need more data to definitely confirm this.

Detailed analyses of the magnitude and duration of rotation for the Tablet conditions are shown in Figure 8. This data illustrates our earlier observation of three main styles of drawing. We also computed the number of major rotations

\section{Label Task}

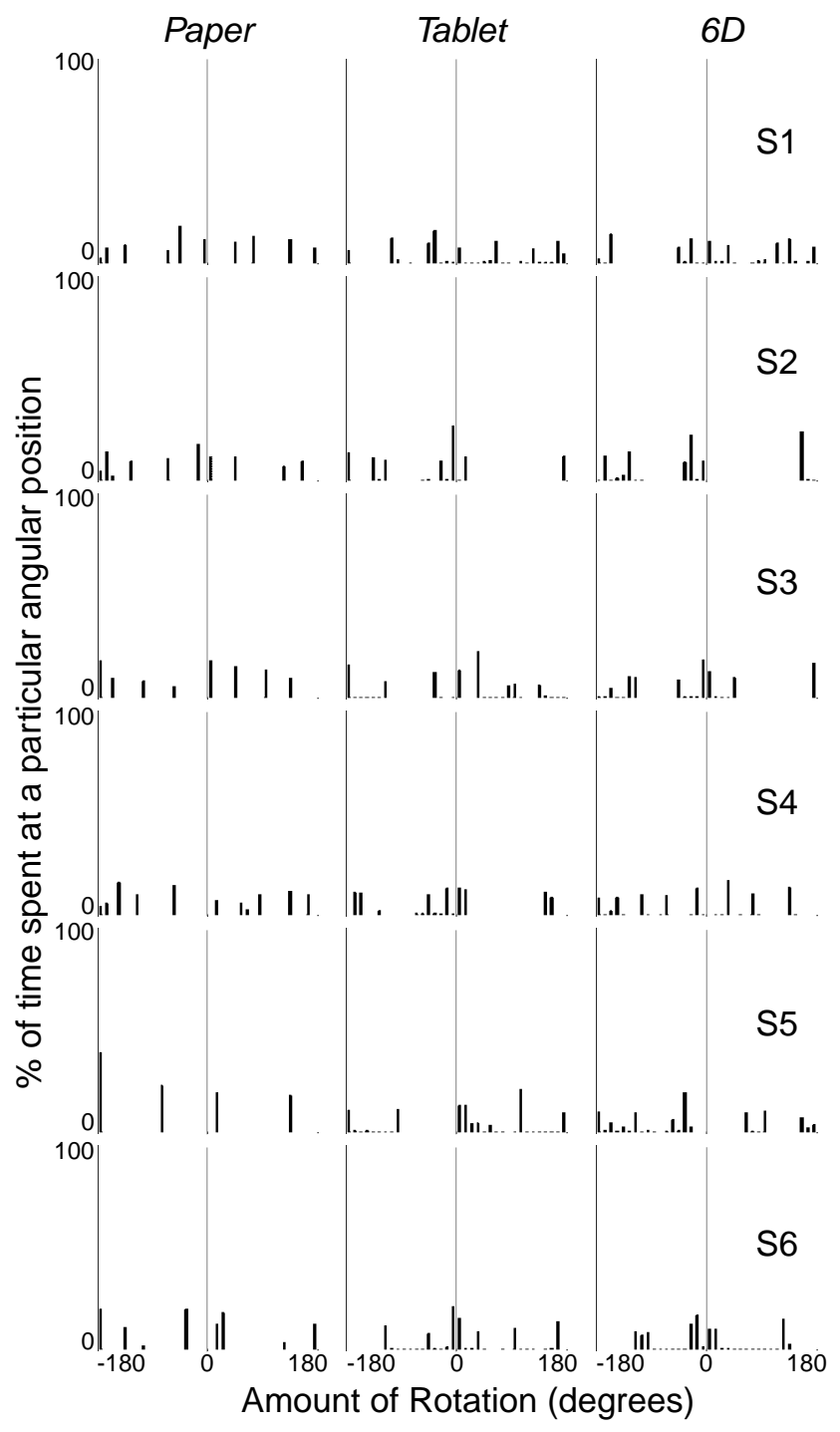

Figure 5: Graphs showing the percentage of time spent by each subject at particular angular positions for the Label task in the Paper, Tablet, and 6D conditions. The faint dotted line in the centre of each graph denotes the "zero" position, where the drawing surface was perpendicular to the subject's body. Negative angles indicate counterclockwise rotation, positive angles indicate clockwise rotation. The angle of rotation is measured about the axis perpendicular to the drawing surface, and is summarized in increments of 10 degrees.

performed by each subject in each task. This number is usually larger than the number of orientations used, which indicates that subjects rotate back and forth between a few primary orientations. Analysis for the $6 D$ conditions shows similar results.

\section{Differences Between Paper, Tablet, and 6D}

In designing this study, we included three conditions to see if the cord on the tablet, and the weight/form-factor of the tablet had any impact on subject's drawing styles and manipulation of the drawing surface. Aside from the few anomalies discussed previously, the only major difference 
Relative to Paper
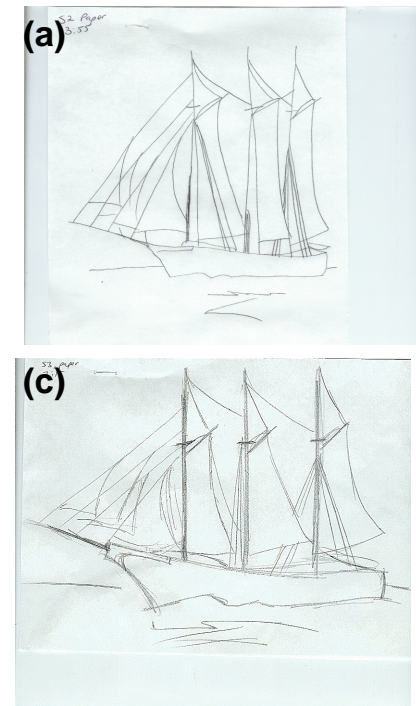

(e)

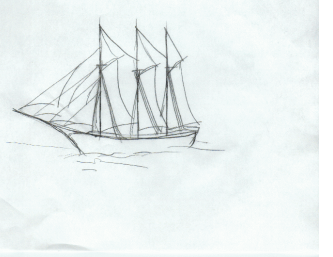

\section{Relative to Table}

(b)

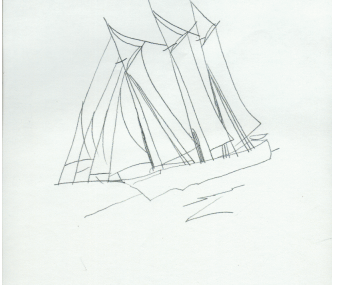

(d)

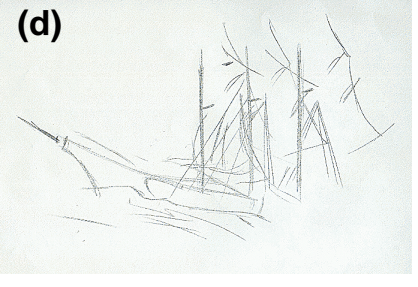

(f)

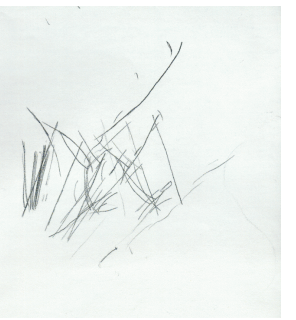

Figure 6. Sample results of exploratory study in the Paper condition with the Ship drawing task. The left column shows the results of the drawing of 3 subjects. The right column shows the respective results relative to the table. This was captured using carbon paper as described in the "Description of Study" section of this paper.

we observed was between the Paper and Tablet/6D conditions for the Label task. As shown in Figure 7, in the Paper condition some subjects performed a series of rotations all in the same direction. We refer to this as the "corkscrew" effect. This "corkscrew" behavior was not observed in the Tablet $/ 6 D$ condition. We believe that the cord on the tablet inhibited this behavior. However, subjects did not complain about this issue and the task completion time for all three conditions were similar - indicating that while different styles of rotation were employed, one was not dramatically better than the other. Also, this effect was not apparent in the Text and the three drawing tasks.

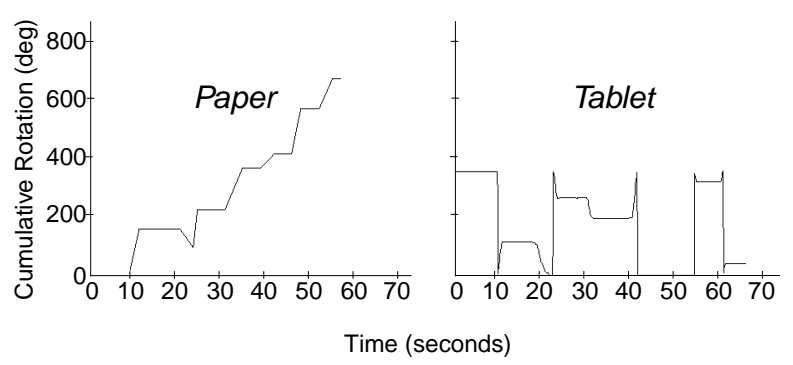

Figure 7: Progression of rotation for the duration of the Label task for Subject \#6 in the Paper and Tablet conditions.
Subjects did not mention, nor did we expect or observe, any adverse effects attributable to the weight/form-factor of the tablet. This could be due to the fact that tablet used was lightweight $(<1$ pound) and fairly thin $(<0.5 \mathrm{inch})$.

\section{Discussion}

While we observed the need for artwork rotation, we can speculate on reasons for not rotating while drawing. If an artist is using another image as a guide to drawing, they may want to keep both images at the same orientation. Rotating the drawing makes it more difficult to copy a nonrotated source image. This "alignment" may be critical when initially copying the basic shape and proportions of

\section{Three Drawing Tasks: Tablet Condition}

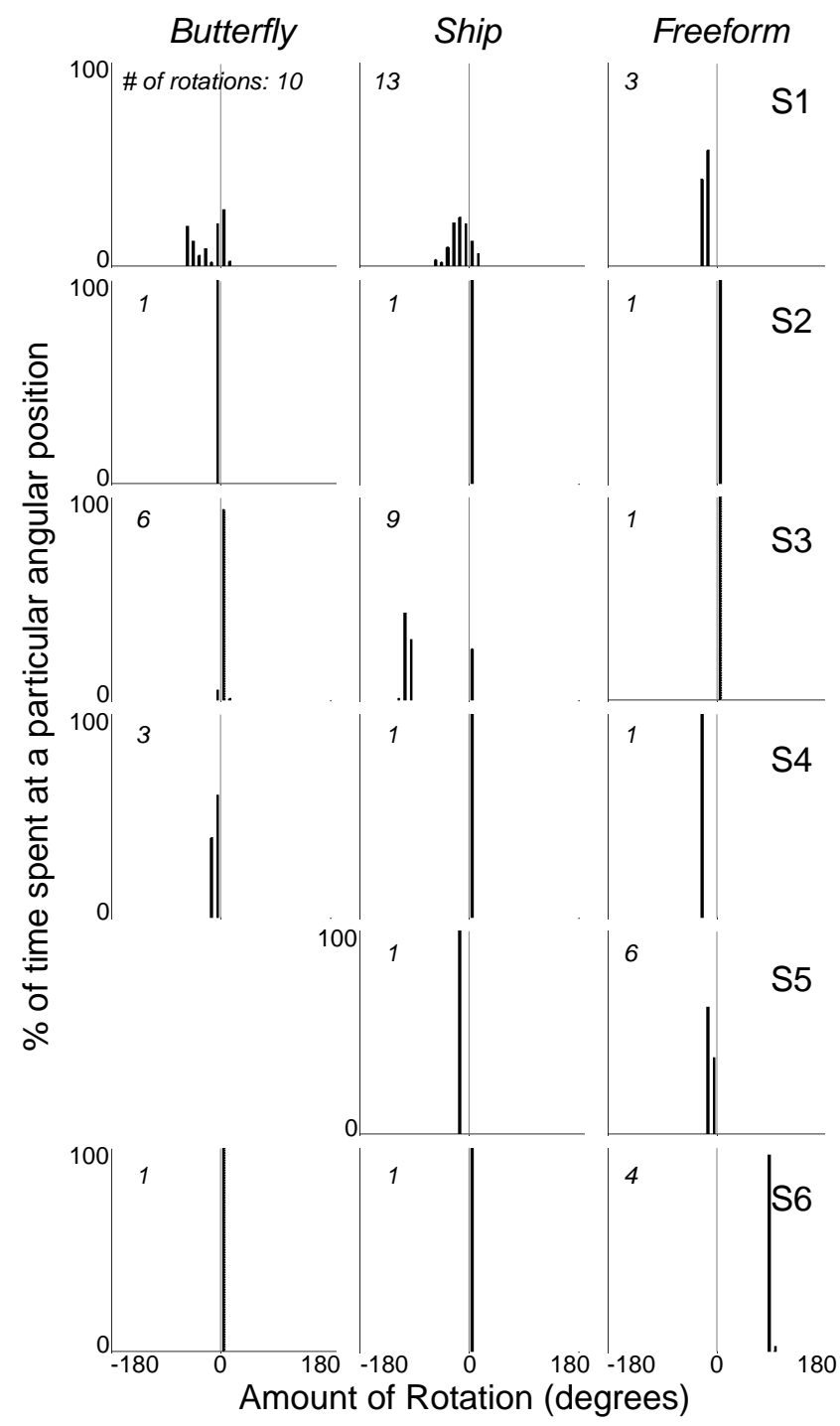

Figure 8: Percent of rotation for the 3 drawing tasks in the Tablet condition. The faint dotted line in the centre of each graph denotes the "zero" position, where the drawing surface was perpendicular to the subject's body. Negative angles indicate counterclockwise rotation, positive angles indicate clockwise rotation. Note that the number of rotations may not be equivalent to the number of bars in the corresponding graph since back and forth rotations between two or more positions would result in a small number of bars but a large number of rotations. Subject \#5's Butterfly task data was lost due to a technical problem. 
an image. Alignment may not be so critical later when shading the drawing. Indeed, our study provided some observations of subjects "aligning" the source image with their drawing. The subject in Figure $2 c$ exhibits this behavior.

Another reason for not rotating is the ability to rotate or adjust one's body rather than rotating the artwork. We observed some subjects turning themselves at an angle to the artwork rather than rotating the artwork. Similarly, if artwork has been rotated to an angle to facilitate physical comfort or drawing in a particular direction, the artist doesn't necessarily have to rotate the artwork back to a normal orientation to evaluate it. The artist can align their head or body with the artwork or not move at all if the angle of rotation is not significant enough to hinder perception of the drawing.

An artist's skill level may also play a factor in rotation. As mentioned earlier, we observed that one subject (S1) who is a professional artist rotated more than the other subjects and was faster and more relaxed. We made a similar observation with a pilot subject who was also a professional artist. Our non-professional artists appeared to be a bit intimidated by our study paraphernalia (drawing on the Wacom tablet/sensor combination, being recorded by an overhead video camera). We suspect than this nervousness might have affected their drawing behavior. We don't have enough data to make any solid conclusions, but we are interested in pursuing this issue.

\section{SUPPORTING ARTWORK ORIENTATION}

Given our exploratory study where we were able to characterize manipulation behavior for drawing tasks, we now consider possible solutions for supporting artwork orientation in the user interface. The primary issue is how do we present, manage, and preserve UI operability while the interface changes orientation.

Imagine using an interactive drawing surface where the traditional GUI user interface is rotated 90 degrees. The menus don't pop-down, they pop sideways, and the text is vertical (e.g., Figure 9a,b). There are similar problems with most GUI elements. We now present a few UI solutions to facilitate artwork orientation.
Separate displays. One option is to provide multiple displays to the user where one display contains the artwork and a second contains the UI controls. In this configuration, the artwork canvas can be oriented independently from the UI controls. This, however, results in larger workspaces and perhaps a bit more clutter. It also results in divided attention issues where the eye and hand must move back and forth from the control display to the artwork display. Also, this approach eliminates the capacity for context-sensitive UIs such as marking menus[10], tool glass and magic lenses[3].

Software canvas rotation. An alternate solution is to allow the artwork canvas to be rotated via the software while the physical display and UI remain fixed [6]. This solution is used in a few commercially available packages but there are several problems with this approach. First, rotating the artwork is generally a computationally intensive task that often introduces annoying delays while drawing. Second, for artwork consisting of rasters of pixels, the aliasing that results when rotating a raster of pixels by anything other than increments of 90 degrees can be annoying. Advances in graphics hardware and texture memory have largely alleviated this problem, however, it is still hard for these technologies to compete with simply rotating the artwork by rotating the display. Thirdly, given that the canvas is the same size as the display, the software rotation will clip portions of the canvas depending on the orientation. Thus, this approach potentially suffers from a great deal of wasted display space.

Do nothing. This sounds like a silly and unfriendly solution but doing nothing is very easy to implement. The idea is that the user will adapt to slight orientation offsets or rotate the display back to the standard orientation to operate the UI controls. We believe this solution will hinder professional artists who want to actively orient their drawing canvas as they draw as well as efficiently issue UI commands.

Rotating User Interface. There may be a need for a UI which is "self righting", or rather, maintains its orientation relative to the user. This is a new approach which we are calling "rotating user interfaces" (RUIs). We now discuss this solution in detail along with design issues.

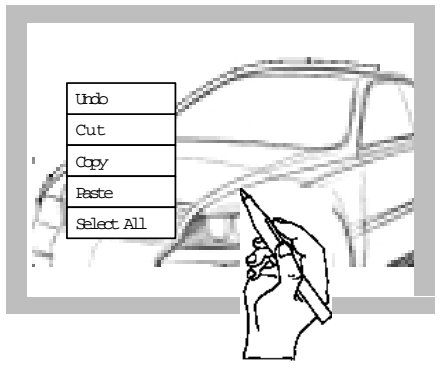

(a)

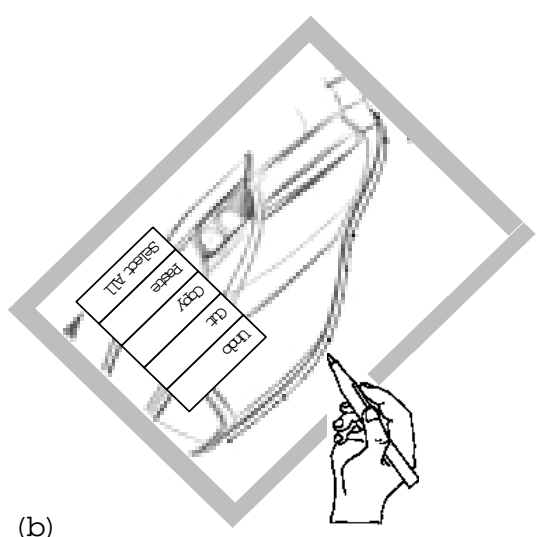

(b)

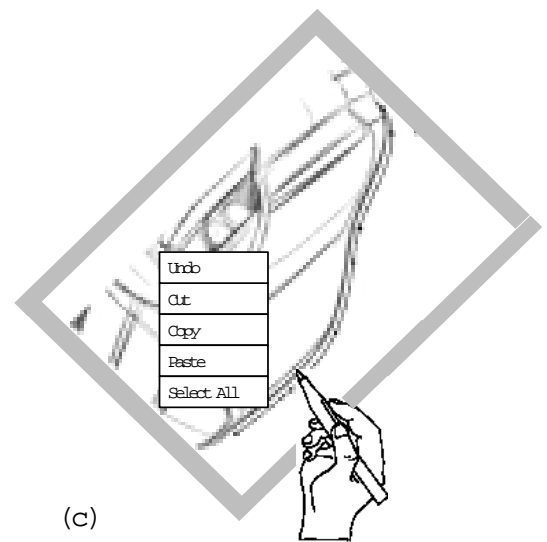

Figure 9: The concept of a rotating UI. a) shows a pen-based computer at normal orientation. Rotating the artwork (b) may give a drawing advantage but the UI becomes hard to use. (c) shows how rotating the UI relative to the user solves the problem. 


\section{ROTATING USER INTERFACES}

We define a rotating user interface as a system where the display rotates around an axis perpendicular to the center of the display. However, the UI remains fixed relative to the user while the artwork rotates. Note that this requires a system where the display can sense its rotation and display the UI so that it remains fixed relative to the user (Figure 9).

RUIs can be discrete or continuous. Discrete RUIs allow the UI to be rotated in discrete steps, typically 0 and 90 degrees. For example, the PenPoint [5] user interface could be rotated 0 degrees for a portrait display orientation or 90 degrees for landscape orientation (however, in this case the artwork also rotates so it is not a RUI by our definition).

\section{Design Issues for Rotating Uls}

Given this simple definition, we have discovered numerous design issues concerning RUIs. One issue is with the rotation sensitive UI components. Some common UI widgets maintain their usability despite severe rotation. We call this property "rotation insensitive." In contrast, some UI widgets are "rotation sensitive" and are severely compromised by rotation. Figure 10 shows examples of both types. A set of color palettes are rotation insensitive. Rotation sensitive widgets are those widgets whose graphics or interaction have some sense of orientation. For example, text and some icon legibility are severely affected by rotation. Other examples are widgets which are directional (e.g., a horizontal slider that is labeled "left" and "right"). Input to a widget can be sensitive to orientation as well. This generally occurs when a widget has no visuals to indicate orientation. For example, marking menus [10] use directional strokes to select menu items from a radial menu without displaying the menu. This runs into problems when the display is rotated. For example, an "up stroke" will select a different item depending on the orientation of the display. Similar problems can occur in UIs that recognize handwriting or gestures.

There are several temporal issues concerning widget rotation. Pop-up UI widgets may be more suitable to RUIs than static widgets. For example, as shown in Figure 9, a pop-up menu need only reorient itself to the current rotation when it is popped up. However, a static widget may have to update itself constantly as the display is being rotated. This may produce annoying flashing or slow system perfor-

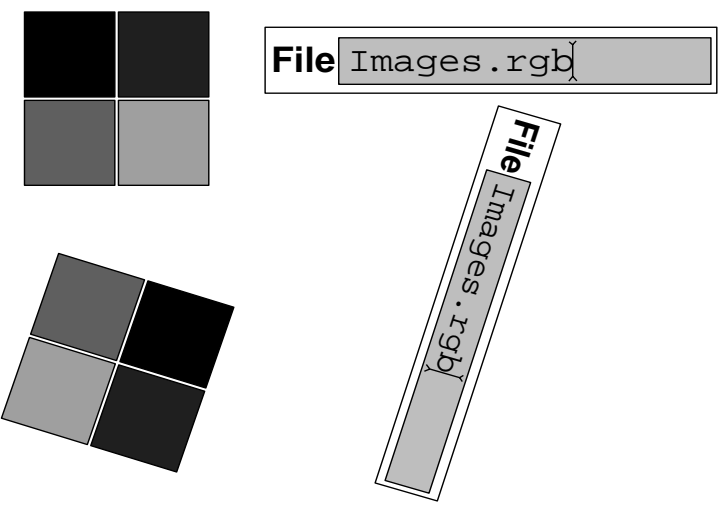

Figure 10. Some widgets are rotation insensitive while others are not. The color pallet on the left is still usable despite the severe rotation. The file name text prompt on the right however becomes very difficult to use at the similar rotation. mance. This problem could possibility be controlled by delaying the reorientation of the static graphics until the user stops rotating. However, this solution may still produce performance delays when the user stops rotating. Note that pop-up widgets may have to address the issues of interactive rotation as well if the display is rotated while the widget is popped up. However, a reasonable simplifying assumption might be that a user will not significantly rotate the display while interacting with a temporary pop-up widget. This assumption requires further investigation.

All of these issues indicate a RUI, at best, would have to support arbitrary rotation of standard GUI widgets. This is extremely problematic because standard GUI toolkits do not support arbitrary rotation transformations of their primitive objects or input events. For example, primitive graphics like text fonts and rectangles are assumed to be nonrotatable. However, 3D graphics toolkits like OpenGL [12] are capable of arbitrary orientation of primitives and could be used as the basis for a RUI toolkit. The research prototype T3 [11] is a UI that is based entirely on transformational 3D graphics primitives and therefore can very easily be adapted to a RUI system.

In addition to being able to rotate graphics primitives, widget layout routines may have to be more sophisticated. Figure 11 shows a case where, when the display is rotated, the widgets cannot just simply rotate but need to reformat themselves to suit the angle of the rectangular display. This will be especially true for GUI designs that use widgets along the perimeter of the display, such as menubars and toolbars. This is yet another issue for a RUI toolkit to address. One possible solution is to design better layout algorithms. Another, perhaps simpler, solution would be only to use widgets which don't track the perimeter of the display (e.g., pop-up menus). These issues require further investigation.

Also, the technology for sensing the rotation of the display/ input system needs to be developed. Current solutions usually require a reference point [1], or complicated mechanical contraptions. Furthermore, this orientation information needs to be fed to the RUI toolkit.

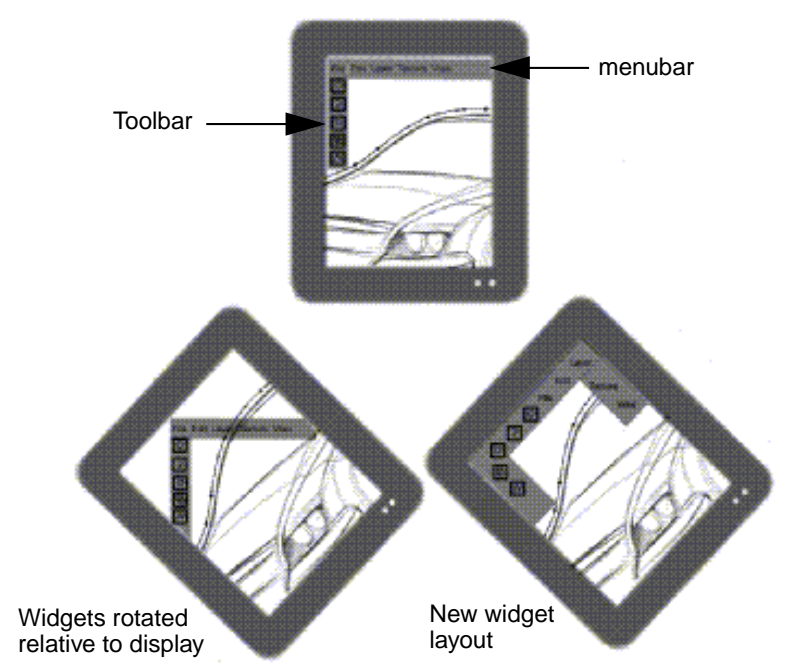

Figure 11. Not only do UI graphics have to be able to draw at arbitrary orientations in a RUI, perimeter widgets like menubars and toolbars may have to adopt different layout schemes depending on the rotation. 
Finally, viewing angle of the display can also be an important issue for RUI systems. Image quality on most flat panel displays (e.g., LCD or TFT displays) changes depending on the user's viewpoint. Thus, a rotatable display would have to be of a type that does not suffer from severe image degradation when rotated.

\section{CONCLUSIONS}

Lightweight, flat displays are inevitable and this relatively new form factor places new requirements on traditional GUI designs. Moreover, the combined display and digitizer tablets will place additional demands on the UI as this configuration will be used like paper due to its affordances. The goal of this research is to anticipate future UI needs and identify related design issues as early as possible given these new input and output configurations.

We knew a priori from everyday experience and the "animator's turntable" (Figure 1), that rotating the artwork while drawing is important. Before embarking on designing a user interface for such rotatable displays, we thought it was important to understand the nature and the way in which the artwork is rotated when drawing. We conducted an exploratory study to investigate the range of behaviors inherent in drawing tasks.

Our study showed a wide range of individual differences when performing typical drawing tasks. However, three general behaviors emerged. In one case, the artwork is oriented once to a comfortable position and subsequently kept static for the duration of the drawing. In the second case, the artwork is oriented once initially, and a small number of adjustments are made while drawing. In the third case, the artwork is adjusted numerous times while drawing.

These general findings indicate the need for freely rotatable drawing surfaces (not ones that have discrete steps such as 45 or 90 degrees). Given the range of rotation sometimes performed by users, a rotation sensitive user interface (RUI) will likely be necessary.

Our work has unearthed the design issue that currently available GUI toolkits are unsuitable, nor can they be easily modified, to support RUIs. We believe a better approach would be to develop a new user interface paradigm and toolkit that supports rotation in its core architecture. This is clearly a challenging user interface toolkit research problem.

Even if we do not consider systems explicitly designed to support the rotation of artwork, the issues presented in this paper have implications for the broader field of mobile, pen-based, and slate computer systems.

\section{ACKNOWLEDGEMENTS}

The original idea for RUIs as embodied in this work came from Jeff Bell and Bill Buxton. We also thank Russell Owen, Thomas Baudel and Yves Guiard for their invaluable comments and assistance, and all the participants in our user study. This work was done under the auspices of the User Interface Research Group at Alias|wavefront.

\section{REFERENCES}

1. Ascension Technology, Inc. The Flock of Birds - Position and Orientation Measurement System. URL: www.ascension-tech.com
2. Athenes, S., "Contribution de la main droite a l'acte graphique chez le scripteur gaucher adulte: une comparaison entre postures 'inversee' et 'non-inversee" (Contribution of the right hand in handwriting for lefthanded adults: a comparison of the 'inverted' and 'non-inverted' postures). Universite de Provence, France. Yves Guiard, thesis advisor, June 1983.

3. Bier, E.A., Stone, M.C., Fishkin, K., Buxton, W. and Baudel, T. (1994). A Taxonomy of See-Through Tools. Proceedings of ACM CHI'94 Conference on Human Factors in Computing Systems, 358-364.

4. Bush, V. (1945) As We May Think. Atlantic Monthly, July, 1945. 101-108.

5. Carr, R., and Shafer, D. The Power of Penpoint. Reading, MA. Addison-Wesley, 1991.

6. Fekete, J., Bizouarn, E., Cournarie, E., Galas, T., and Taillefer, F. (1995) TicTacToon: A Paperless System for Professional 2D Animation. Proceedings of the ACM SIGGRAPH '95, 79-89.

7. Fitzmaurice, G., Ishii, H., and Buxton, W. (1995). Bricks: Laying the Foundations for Graspable User Interfaces. Proceedings of the ACM CHI'95 Conference on Human Factors in Computing Systems,442449.

8. Guiard, Y. (1987). Asymmetric division of labor in human skilled bimanual action: The kinematic chain as a model. Journal of Motor Behavior, 19, 486-517.

9. Hinckley, K. (1997). Haptic Issues for Virtual Manipulation. Ph.D. dissertation, University of Virginia, Dept. of Computer Science.

10. Kurtenbach, G., and Buxton, W. (1993). The limits of expert performance using hierarchical marking menus. Proceedings of CHI '93 Conference on Human Factor in Computing, 482-48.

11. Kurtenbach, G., Fitzmaurice, G., Baudel, T., and Buxton, W. (1997). The Design of a GUI Paradigm based on Tablets, Two-hands, and Transparency. Proceedings of the ACM CHI'97 Conference on Human Factors in Computing Systems, 35-42.

12. Neider, J., Davis, T., and Woo, M. (1993). OpenGL programming guide. Addison-Wesley.

13. Rosenbaum, D. A., van Heugten, C. M., and Calwell, G. E. (1996). From cognition to biomechanics and back: The end-state comfort effect and the middle-isfaster effect. Acta Psychologica, 94, 59-85.

14. Sutherland, I. E. (1963). Sketchpad: A man-machine graphical communication system. AFIPS Conference Proceedings, 23, 329-346.

15. Van Sommers, P. (1984). Drawing and Cognition: Descriptive and experimental studies of graphic production processes, Cambridge, England. Cambridge University Press.

16. Wacom Technology Inc., ArtZ II 6X8 tablet. PL-300 display tablet. URL: www.wacom.com. 